\title{
Actin fence therapy with exogenous V12Rac1 protects against acute lung injury
}

\author{
Galina A. Gusarova, ${ }^{1}$ Shonit R. Das, ${ }^{1}$ Mohammad N. Islam, ${ }^{1}$ Kristin Westphalen, ${ }^{1}$ Guangchun Jin, ${ }^{1}$ \\ Igor O. Shmarakov, ${ }^{2}$ Li Li, ${ }^{1}$ Sunita Bhattacharya, ${ }^{1,3}$ and Jahar Bhattacharya ${ }^{1,4}$ \\ 'Lung Biology Laboratory, Division of Pulmonary, Allergy, and Critical Care Medicine, Department of Medicine, ${ }^{2}$ Department \\ of Medicine, ${ }^{3}$ Department of Pediatrics, and ${ }^{4}$ Department of Physiology and Cellular Biophysics, Vagelos College of \\ Physicians and Surgeons of Columbia University, New York, New York, USA.
}

High mortality in acute lung injury (ALI) results from sustained proinflammatory signaling by alveolar receptors, such as TNF- $\alpha$ receptor type 1 (TNFR1). Factors that determine the sustained signaling are not known. Unexpectedly, optical imaging of live alveoli revealed a major TNF- $\alpha-$ induced surge of alveolar TNFR1 due to a $\mathrm{Ca}^{2+}$-dependent mechanism that decreased the cortical actin fence. Mouse mortality due to inhaled LPS was associated with cofilin activation, actin loss, and the TNFR1 surge. The constitutively active form of the GTPase, Rac1 (V12Rac1), given intranasally (i.n.) as a noncovalent construct with a cell-permeable peptide, enhanced alveolar filamentous actin (F-actin) and blocked the TNFR1 surge. V12Rac1 also protected against ALIinduced mortality resulting from i.n. instillation of LPS or of Pseudomonas aeruginosa. We propose a potentially new therapeutic paradigm in which actin enhancement by exogenous Rac1 strengthens the alveolar actin fence, protecting against proinflammatory receptor hyperexpression, and therefore blocking ALI.

\section{Authorship note: SRD is deceased.}

Conflict of interest: The authors have declared that no conflict of interest exists.

Copyright: (ㄷ) 2021, Gusarova et al. This is an open access article published under the terms of the Creative Commons Attribution 4.0 International License.

Submitted: December 17, 2019

Accepted: February 11, 2021

Published: March 22, 2021

Reference information: /CI Insight. 2021;6(6):e135753.

https://doi.org/10.1172/jci.

insight.135753.

\section{Introduction}

Life-threatening tissue injury to critical organs occurs as a result of host-pathogen interactions involving proinflammatory receptors. In the lung, the resulting inflammation underlies acute lung injury (ALI), which can lead to the acute respiratory distress syndrome (ARDS), a condition that is associated with high mortality (1). Pharmacological therapies are not available for ALI but are required in order to stem disease progression.

ALI due to inhaled Gram-negative bacteria occurs through initiating and progressive phases. In the initiating phase, inhaled pathogens ligate TLRs on macrophages and the alveolar epithelium $(2,3)$, causing release of proinflammatory cytokines, such as TNF- $\alpha$, that ligate alveolar epithelial receptors (4). Crosstalk with the endothelium follows (5), and chemoattractants, such as IL-8, activate inflammatory cell recruitment (6). In the progressive phase, recruited and resident immune cells continue to secrete cytokines, thereby sustaining the inflammatory response $(7,8)$. The extent to which the initiating mechanisms continue to enhance the progressive phase of the response remains unclear. Here, we address this issue in the context of the interactions of TNF- $\alpha$ with its alveolar receptor, TNFR1.

Studies from multiple cell types indicate that, following ligation, TNFR1 - a transmembrane protein - sheds its ectodomains (9-11). The sheddase, ADAM-17, mediates the shedding (10), which is protective since shedding inhibition augments lung inflammation (9). The shed domains are not recycled by the cell from which the shedding occurred (12). Brefeldin A, the inhibitor of protein trafficking to the Golgi, abrogated TNFR1 receptor mobilization and decreased TNFR1 abundance on the cell surface, indicating that membrane replenishment of TNFR1 occurs by receptor trafficking from storage pools in the Golgi (11, 13-15). The replenishment is translation independent $(13,16)$. However, the extent and the time course of TNFR1 replenishment in the alveolar epithelium remain unknown.

In this regard, the regulatory role of cortical actin remains unclear. Cortical actin is the layer of filamentous actin (F-actin) that forms a network adjacent to the plasma membrane (PM). Although cortical actin is known to form a "fence" against trafficking of vesicles and receptors to the PM (17, 18), dynamic data from live alveoli - detailing the role of the actin fence in TNFR1 expression - are lacking. Here, we address these issues by application of confocal microscopy of the live alveolar epithelium. Our goal was to 
determine strategies by which fence enhancement might impede alveolar receptor display and, therefore, ALI pathogenesis. We show for the first time, to our knowledge, that loss of the fence causes a proinflammatory surge of TNFR1 expression and that the fence is a druggable target for mitigating ALI.

\section{Results}

F-actin determines alveolar TNFR1 display. Optical access to the live alveolar epithelium of mouse lung provided an opportunity for dynamic evaluation of the role of the F-actin fence as a determinant of epithelial TNFR1 expression. By real-time confocal microscopy of pulmonary alveoli, we determined fluorescence expressions of TNFR1 in the alveolar epithelium in terms of a fluorescent mAb that detects TNFR1 ectodomains (9) (Figure 1A). We confirmed that the TNFR1 immunofluorescence was on the cell surface, since it was eliminated by alveolar injection of a cell-impermeable fluorescence quenching agent. Our procedures did not label TNFR1 on the capillary endothelium adjacent to the epithelium (Supplemental Figure 1A; supplemental material available online with this article; https://doi.org/10.1172/jci.insight.135753DS1). Moreover, costaining with the lamellar body marker lysotracker red (LTR) revealed TNFR1 expression on the type 1 epithelial cells (AT1) and no detectable expression of TNFR1 on the type 2 (AT2) (Supplemental Figure 1B).

To determine the dynamics of epithelial TNFR1 expression, we sequentially microinjected the anti-TNFR1 mAb TNF- $\alpha$ (or PBS) in each alveolar field, and we then repeated the anti-TNFR1 Ab for a second time (Figure 1A). The first mAb injection marked the baseline TNFR1 expression on the alveolar epithelium. Alveolar microinjection of TNF- $\alpha$ rapidly induced a time-dependent decrease of TNFR1 immunofluorescence (Figure 1A), affirming our previous findings that TNF- $\alpha$ causes shedding of TNFR1 ectodomains (9). However, in the same alveoli that had undergone receptor shedding, the second $\mathrm{mAb}$ injection revealed marked enhancement of TNFR1 reexpression (Figure 1A).

In separate experiments, we gave the second $\mathrm{mAb}$ injection at different time points to further delineate the time course of TNFR1 reexpression. Our findings indicate that, within 10 minutes after the TNF- $\alpha-$ induced shedding, TNFR1 expression was similar to baseline (Figure 1B), indicating that the receptor was rapidly replaced. Subsequently, there was a surge of TNFR1 reexpression that, on average, reached a peak at 1 hour and returned to baseline at 4 hours (Figure 1B).

To determine the effects of the cytosolic $\mathrm{Ca}^{2+}$ on F-actin and TNFR1 expression, we microinfused TNF- $\alpha$ in alveoli expressing a transfected F-actin probe (5). At baseline, fluorescence of $\mathrm{Ca}^{2+}$ and $\mathrm{F}$-actin were steady for at least 20 minutes (Figure 1C), ruling out photobleaching as an artefact. Alveolar microinjection of TNF- $\alpha$ rapidly increased $\mathrm{Ca}^{2+}$, while concomitantly decreasing F-actin (Figure 1C). To inhibit the $\mathrm{Ca}^{2+}$ response, we gave alveolar microinjection of the $\mathrm{Ca}^{2+}$ chelator, BAPTA-AM, and then determined responses after 30 minutes. BAPTA-AM blocked the TNF- $\alpha$-induced cytosolic $\mathrm{Ca}^{2+}$ increase, the F-actin decrease, and the TNFR1 surge (Figure 1D). PBS pretreatment had no effect. These first dynamic quantifications of actin in live alveoli indicated a strong effect of the cytosolic $\mathrm{Ca}^{2+}$ on F-actin and TNFR1 expression.

To depolymerize F-actin by a receptor-independent mechanism, we gave an alveolar microinjection of the actin depolymerizing agent cytochalasin $\mathrm{D}(\mathrm{cytD})$ and determined the TNFR1 response after 60 minutes (19). CytD exposure markedly enhanced TNFR1 expression (Figure 1E). Thus, even in the absence of TNF- $\alpha$, actin depolymerization with CytD was sufficient to induce the TNFR1 surge. Taken together, these findings affirmed that F-actin constitutively inhibited alveolar TNFR1 expression, and that decrease of F-actin caused the TNFR1 surge.

TNF- $\alpha$ causes calcineurin-dependent alveolar TNFR1 expression. To determine mechanisms downstream of the $\mathrm{TNF}-\alpha$-induced cytosolic $\mathrm{Ca}^{2+}$ increase, we considered the role of calcineurin $(\mathrm{Cn})$, which is a $\mathrm{Ca}^{2+}$ sensor. $\mathrm{Cn}$ is a $\mathrm{Ca}^{2+}$-calmodulin dependent protein phosphatase that contains the catalytic $\mathrm{CnA}(\alpha, \beta$, and $\gamma$ isoforms) and the $\mathrm{Ca}^{2+}$-sensing $\mathrm{CnB}$ subunits $(20,21)$. In $\mathrm{CnA} \beta$-null mice, the $\beta$ isoform of $\mathrm{CnA}$ is disrupted, inhibiting $\mathrm{Cn}$ 's phosphatase activity (22). Optical imaging revealed that the TNF- $\alpha$-induced F-actin decrease and the accompanying TNFR1 surge were absent in CnA $\beta$-null mice (Figure 2A and Supplemental Figure 2A). Moreover, the $\mathrm{Cn}$ inhibitor FK-506 blocked the TNFR1 surge (Supplemental Figure 2B). These findings mechanistically implicated $\mathrm{Cn}$ in the TNFR1 responses. $\mathrm{Cn}$ dephosphorylates, hence, activates the actin-severing protein cofilin $(23,24)$. To evaluate the role of $\mathrm{Cn}$ in cofilin activation, we carried out immunoblots on lysates of lungs of WT and CnA $\beta$-null mice. Our findings indicated that levels of phosphorylated (inactive) cofilin were higher in CnA $\beta$-null than WT mice (Figure 2B). Moreover, 4 hours after intranasal (i.n.) instillation of the TNF- $\alpha$, cofilin dephosphorylation occurred in WT but not CnA $\beta$-null mice (Figure 2B). We interpret from these findings that TNF- $\alpha$-induced $\mathrm{Cn}$ activation led to cofilin dephosphorylation. 

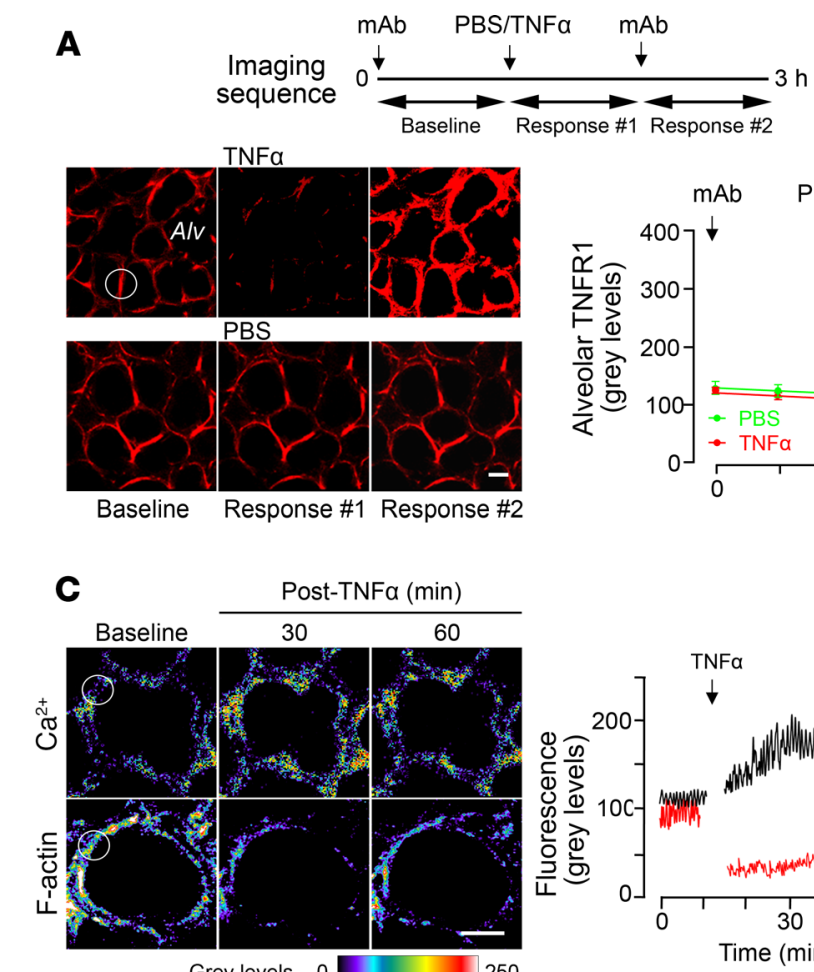

Grey levels
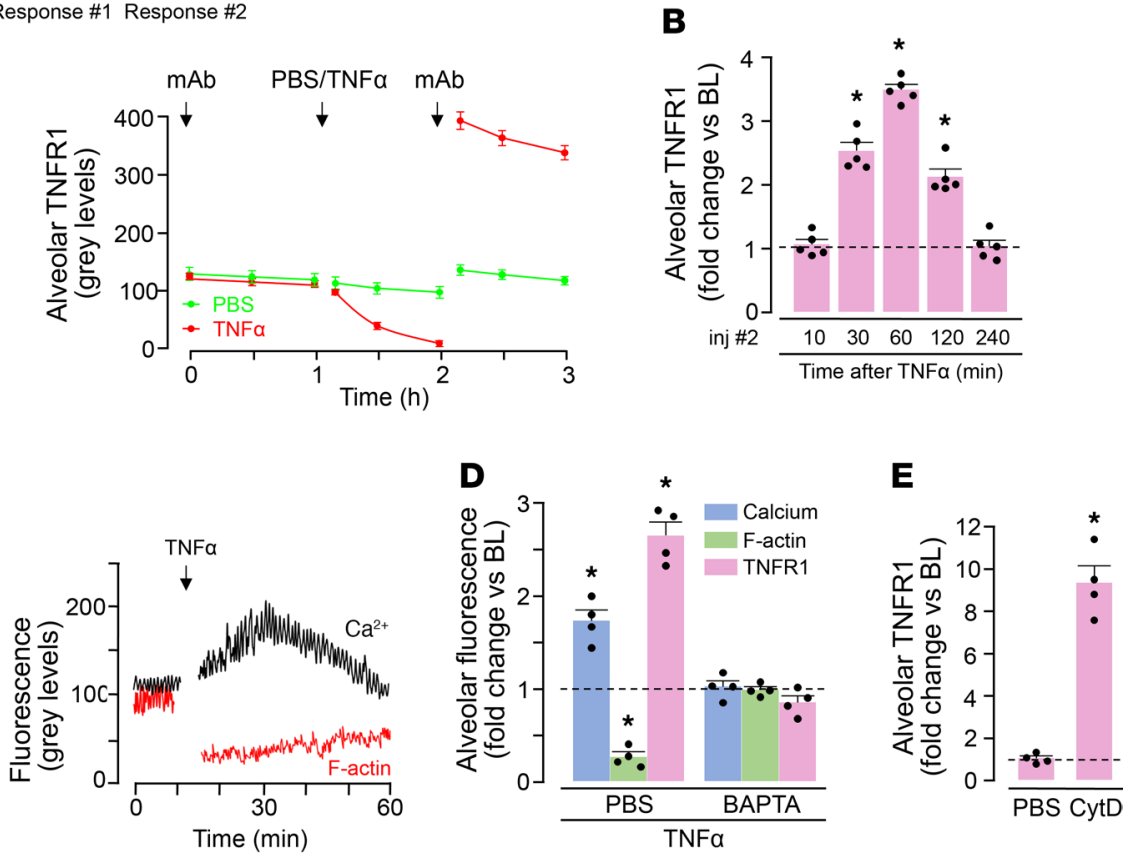

Figure 1. F-actin determines surge in alveolar TNFR1 expression. (A) Confocal images of live alveoli show TNFR1 expression (red). Images were obtained at baseline $(B L)$ and after indicated alveolar microinfusions (arrows in sketch and plotted data). TNFR1 fluorescence was quantified as gray levels at a region of interest (ROI, dashed circle). The gray levels are shown as the time line plot with the time points of the microinjections marked by arrows. Each plotted point shows the average for 15 ROI from 5 alveoli of a single lung (mean \pm SEM). Replicated in 3 lungs. Scale bar: $20 \mu \mathrm{m}$. Alv, alveolus. (B) Data show group responses to alveolar microinjection of TNF- $\alpha$. The response time points are as indicated. For each bar, $n=5$. ${ }^{*} P<0.05$ versus baseline (dotted line). (C) Confocal images of live alveoli show pseudocolored fluorescence of cytosolic $\mathrm{Ca}^{2+}$ and $\mathrm{F}$-actin, as determined by fluorescence of Fluo 4 and Lifeact, respectively. The tracings show alveolar epithelial responses to alveolar microinfusion of TNF- $\alpha$ in a single lung. Replicated 5 times in each of 3 lungs. For the tracings, in the baseline image, ROls were selected at gray levels of 80-120 to accommodate detection of subsequent increases or decreases of fluorescence within the dynamic range of the imaging system. Scale bars: $10 \mu \mathrm{m}$. ( $\mathbf{D}$ and $\mathbf{E}$ ) Data are whole-image gray levels normalized to the corresponding baseline (dashed lines). Responses shown are to alveolar microinjection of TNF- $\alpha$ after 30 minutes (D). The responses to cytochalasin $D(C y t D)$ were obtained after 60 minutes (E). Data are presented as mean \pm SEM. Each dot shows data for a single lung. For each bar, $n=4$ (C and E) or 5 (D) lungs. ${ }^{*} P<0.05$ versus baseline (dotted lines) using ANOVA with Bonferroni correction.

To further explore the role of cofilin, we transfected the alveolar epithelium with plasmids to express WT cofilin $(p \mathrm{WT})$, or cofilin mutants that cannot be phosphorylated ( $p \mathrm{~S} 3 \mathrm{~A})$, or are constitutively phosphorylated $(p \mathrm{~S} 3 \mathrm{E})$. Therefore, these mutants are, respectively, constitutively active or inactive (Supplemental Figure 3) (25-27). As compared with $p \mathrm{WT}$-expressing epithelium, baseline F-actin was lower in $p \mathrm{~S} 3 \mathrm{~A}$-expressing but higher in $p \mathrm{~S} 3 \mathrm{E}$-expressing epithelium (Figure $2 \mathrm{C}$ ). Alveolar TNF- $\alpha$ microinjection decreased $\mathrm{F}$-actin in $p \mathrm{~S} 3 \mathrm{~A}$-expressing but not in $p \mathrm{~S} 3 \mathrm{E}$-expressing epithelium (Figure $2 \mathrm{C}$ ). This lack of effect was not due to detection failure, since the actin depolymerizing agent latrunculin B decreased F-actin in $p \mathrm{~S} 3 \mathrm{E}$-expressing epithelium (Figure $2 \mathrm{C}$ ). The TNF- $\alpha$-induced TNFR1 surge was greater in $p \mathrm{~S} 3 \mathrm{~A}$-expressing than WT-expressing epithelium, but it blocked in $p \mathrm{~S} 3 \mathrm{E}$-expressing epithelium (Figure $2 \mathrm{D}$ ). These findings indicate that active, namely dephosphorylated, cofilin was required for the TNF- $\alpha$-induced TNFR1 surge. Taken together, our findings indicate that a major effect of TNF- $\alpha$ was to activate $\mathrm{Cn}$, which then led to cofilin dephosphorylation. As a consequence, epithelial F-actin decreased, resulting in the TNFR1 surge.

Delivery of exogenous Rac1 mutants modifies the actin cytoskeleton in alveolar epithelium. The GTPase Rac1 phosphorylates p21-activated kinase, leading to LIM-kinase-dependent cofilin phosphorylation and, therefore, F-actin stabilization (28-31). To evaluate the therapeutic potential of these mechanisms, we developed noncovalent TAT-linked conjugates with His-V12Rac1 and His-N17Rac1, which are constitutively active and inactive mutants of Rac1, respectively (32). We engineered the constructs to be unstable at low $\mathrm{pH}$ to enable intracellular separation of TAT from the cargo protein - therefore, to retain the protein in the cytosol. Accordingly, when we labeled TAT and V12Rac1 with different fluorophores 
A

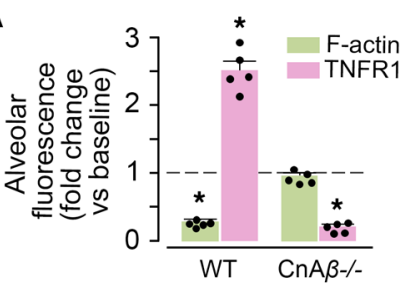

B

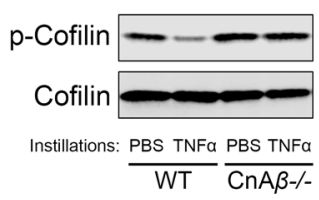

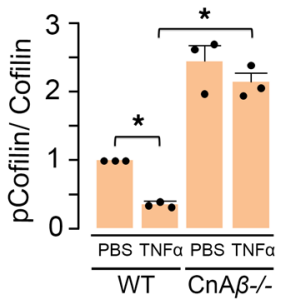

C
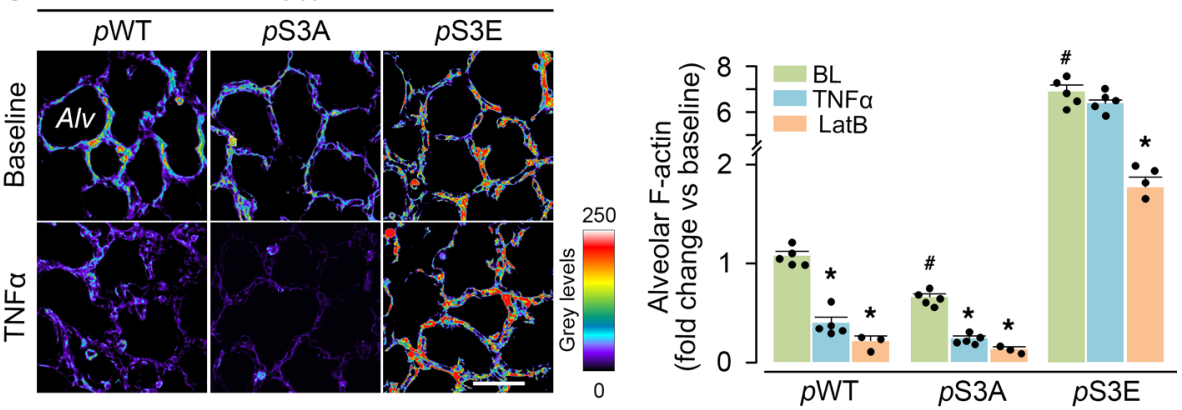

D
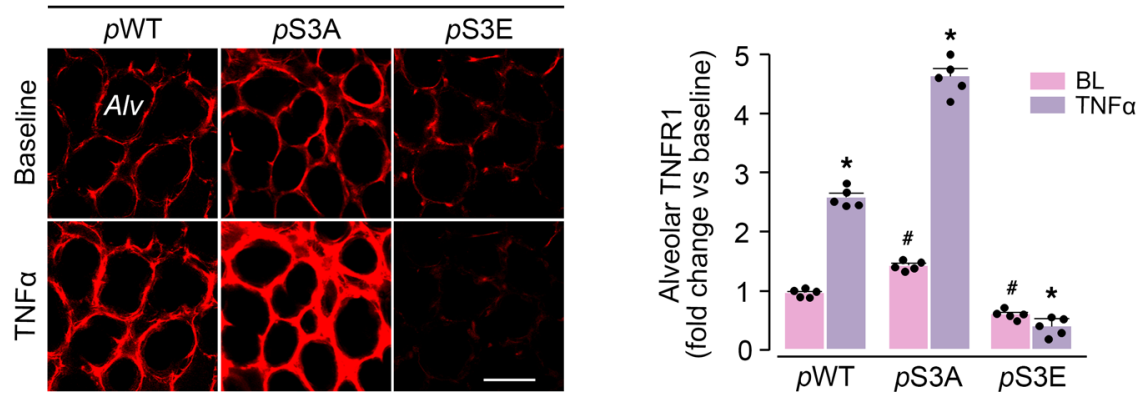

Figure 2. Calcineurin-dependent alveolar TNFR1 expression. (A) Alveoli were optically imaged to quantify fluorescence of F-actin (Lifeact) and TNFR1 (immunofluorescence) in WT and calcineurin-A $\beta$-null ( $\left.C n A \beta^{-1-}\right)$ mice. Data were obtained as whole-image fluorescence (gray levels above background) at baseline (dashed line) and 30 minutes after alveolar microinfusion of TNF- $\alpha$. Microinjections of anti-TNFR1 Ab for TNFR1 detection were given prior to and 30 minutes after TNF- $\alpha$. Data are presented as \pm SEM. $n=5$ lungs for each group. ${ }^{*} P<0.05$ compared with baseline using ANOVA with Bonferroni correction. (B) Immunoblots and densitometry of lung lysates obtained 4 hours after i.n. TNF- $\alpha$. Replicated 3 times. ${ }^{*} P<0.05$ as indicated. (C and D) Cofilin transfections were for WT plasmid $(p W T$ ), and for constitutively active ( $p S 3 A)$, or inactive ( $p S 3 E$ ) cofilin mutants. LatB, latrunculin B. Images in C show alveolar F-actin in terms of rhodamine-phalloidin fluorescence at baseline (upper panel) and 30 minutes after alveolar injection of TNF- $\alpha$ (lower panel). The images in $\mathbf{D}$ were obtained at baseline after the first microinjection of anti-TNFR1 Ab (upper panel). A second Ab microinjection for TNFR1 detection was given 30 minutes after TNF- $\alpha$ microinjection (lower panel). The data in $\mathbf{C}$ and D are quantifications of whole-image fluorescence (mean $\pm \mathrm{SE}$ ). Alv, alveolus. Scale bars: $50 \mu \mathrm{m}$. Each dot shows data for a single lung. $n=5$ for all groups, except LatB ( $n=3$ for $p$ WT and $p S 3 A, n=4$ for $p S 3 E)$. ${ }^{*} P<0.05$ versus baseline for the same group using 2-tailed $t$ test; ${ }^{~} P<0.05$ baseline for the $p$ WT group using ANOVA with Bonferroni correction.

and injected the construct by alveolar microinjection, we could detect rapid entry of TAT-V12Rac1 in the alveolar epithelium (Figure 3A). Subsequently, TAT fluorescence progressively decreased (Figure 3A), indicating that TAT exited from the epithelial cytosol, while V12Rac1 remained. We affirmed that the spatial distribution of V12Rac1 fluorescence in the epithelium matched that of the cytosolic dye Calcein Red (Supplemental Figure 4A), and we affirmed that the cell-impermeable fluorescence quencher Trypan blue (TB) failed to diminish V12Rac1 fluorescence (Supplemental Figure 4, A and B).

To determine whether the TAT-protein constructs entered the endothelium of adjoining capillaries, we gave alveolar microinjections of TAT-V12Rac1 in which V12Rac1 was fluorophore tagged. Then, we loaded the alveolar epithelium and the endothelium with Calcein Red. Alveolar microinjection of the detergent saponin eliminated epithelial but not endothelial cytosolic fluorescence. Thus, the endothelial PM was intact and no V12Rac1 fluorescence was evident in the endothelial cytosol (Supplemental Figure 4, A and B). We conclude that the construct did not cross the alveolar barrier to enter the endothelium. 
Alveolar microinjection of TAT-V12Rac1 induced rapid increase of epithelial F-actin that was sustained for at least 4 hours (Figure 3B). Although baseline F-actin was higher in AT2 than AT1 $(P<0.01)$ (33), V12Rac1 induced a greater relative increase of F-actin in AT1 (Supplemental Figure 5). By contrast, the constitutively inactive form of Rac1, N17Rac1, which was also internalized by the epithelium, failed to increase actin (Figure 3B). The TNF- $\alpha$-induced TNFR1 surge was absent in TAT-V12Rac1-loaded epithelium but present in epithelium loaded with TAT-N17Rac1 (Figure 3B). These findings indicate that epithelial loading with V12Rac1 increased F-actin, inhibiting the TNF- $\alpha$-induced TNFR1 surge.

To determine the extent to which these responses occurred at a whole-organ level, we carried out immunoblots on lung lysates 4 hours after i.n. instillation of the TAT-linked constructs. Our findings indicated that, within 4 hours, TAT-V12Rac1 - but not TAT-N17Rac1 — increased F-actin, while decreasing G-actin (Figure 3C) and concomitantly increasing cofilin phosphorylation (Figure 3D). To determine longer-term effects, we gave i.n. instillations of the TAT constructs. Then, after 24 hours, we immunoprecipitated the Rac1 mutants from lung lysates using anti-His Ab. Both His-tagged proteins were detectable in immunoblots (Figure 3E), indicating that exogenous Rac1 mutants remained in the lung for at least 24 hours after cell internalization. Taking the imaging and the immunoblot data together, our findings indicate that epithelial loading with V12Rac1 increased F-actin, inhibiting the TNF- $\alpha$-induced TNFR1 surge.

Protective effects of alveolar F-actin enhancement on ALI outcomes. To determine the role of alveolar F-actin in a mouse model of ALI, we gave i.n. LPS at lethal dose 0 (LD0), at which there is no mouse mortality $(7,34)$, and then determined responses after 24 hours. In optically imaged alveoli, LPS increased epithelial TNFR1 expression (Figure 4A). Concomitantly, there was alveolar inflammation, as indicated by alveolar neutrophil entry (Figure 4A). By contrast, i.n. instillation of TAT-V12Rac1 30 minutes prior to LPS, but not of TATN17Rac1, blocked the increase of TNFR1 expression, as well as the alveolar inflammation (Figure 4A). LPS induced the expected ALI responses after 24 hours - namely, increased leukocytes in the bronchoalveolar lavage (BAL) (Figure 4B) and increased alveolar permeability to intravascularly injected albumin (Figure 4C).

To determine global lung responses, we carried out in situ biotinylation assays to determine cell-surface expression of TNFR1, as well as assays of whole lung lysates for cofilin phosphorylation, F-actin, and IкB. These studies indicated that LPS decreased cofilin phosphorylation (Figure 4D), as well as F-actin, in 24 hours (Figure 4E) while increasing surface TNFR1 expression (Figure 4F). By contrast, pre-LPS i.n. instillation of TAT-V12Rac1, but not of TAT-N17Rac1, stabilized F-actin for 24 hours (Figure 4E) and markedly abrogated the LPS-induced enhancement of TNFR1 expression. Since NF- $\kappa B$ activation causes alveolar inflammation, we confirmed that LPS-induced IкB degradation (Figure 4G). TAT-V12Rac1, but not TAT-

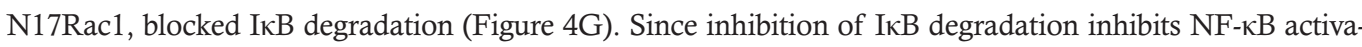
tion, we interpret that V12Rac1-induced actin enhancement inhibited NF-kB activation. Taking the imaging and global data together, our findings indicate that the LPS-induced proinflammatory effect of epithelial TNFR1 hyperexpression was sustained for at least 24 hours and that these responses were inhibited by epithelial incorporation of V12Rac1.

To determine the effects of the Rac1 constructs on LPS-induced mortality, we exposed mice to a lethal LPS dose, which caused robust increases of leukocyte counts and protein concentration in the BAL (Supplemental Figure 7A), depletion of surfactant phospholipids in the BAL (Figure 5A and Supplemental Figure 7B), loss of lung compliance (Figure 5B), and approximately 80\% mortality in 3-4 days (Figure 5C). Pretreatment with i.n. TAT-V12Rac1 30 minutes prior to LPS instillation protected against mortality (Supplemental Figure 6). To evaluate post-ALI therapeutic efficacy, we gave the constructs 4 or 24 hours after LPS. In the 4-hour group, V12Rac1 mitigated LPS-induced ALI as indicated by recovery of lung compliance and BAL surfactant phospholipids (Figure 5, A and B), and a 15\% mortality in 3 days (Figure 5C). In the group given TAT-V12Rac1 24 hours after LPS, evaluation of ALI after a further 24 hours indicated marked reduction of lung water and BAL leukocytes (Figure 5, D and E), as well as a reduction of mortality (Figure 5F). TAT-N17Rac1 was without effect (Figure 5F). The reduction of mortality between the 4- and 24-hour groups was not statistically significant. These findings indicate that, given 30 minutes before, 4 hours after LPS, or 24 hours after LPS, TAT-V12Rac1 protected against ALI.

To determine whether the i.n.-delivered constructs entered the capillary endothelium, we gave mice i.n. LPS at a LD, or PBS, followed 4 hours later with i.n. TAT-V12Rac1-GFP. After another hour, we freshly isolated cells from harvested lungs; we then carried out flow cytometry analyses on the cells. These analyses indicated that V12Rac1 was taken up in the epithelium but not the endothelium (Supplemental Figure 7C). Thus, while the imaging data indicate there was no endothelial uptake of the construct under baseline 
A

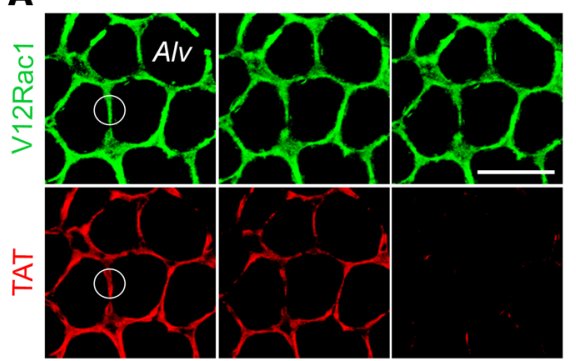

30

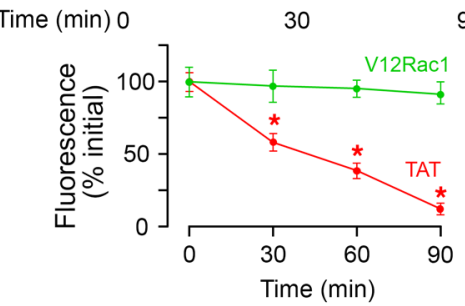

B PBS $\quad$ V12Rac1 N17Rac1

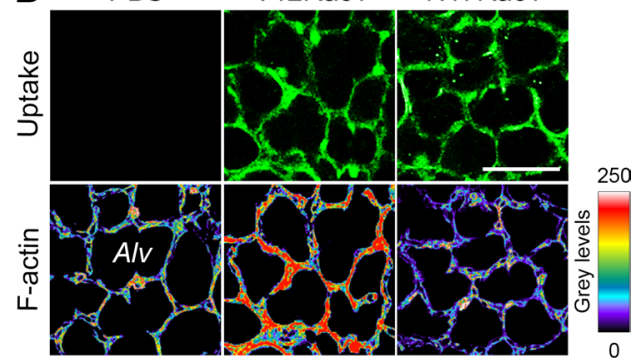

V12Rac1 N17Rac1

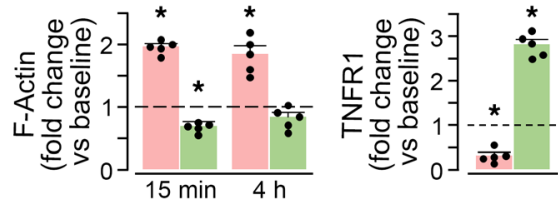

$\mathbf{E}$

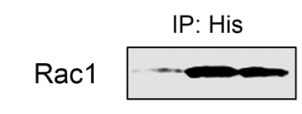

Instillations: PBS V12 N17

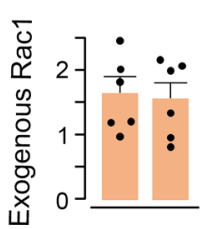

Figure 3. Exogenous delivery of Rac1 mutants modifies the actin cytoskeleton in alveolar epithelium. (A) Confocal images and the plots show changes in alveolar fluorescence of differentially tagged TAT and V12Rac1. The tagging was done prior to conjugation. The plot shows data from ROls (dotted circles) for 10 alveoli of a single lung (mean $\pm \mathrm{SEM}$ ). Replicated in 3 lungs. Scale bar: $50 \mu \mathrm{m} .{ }^{*} P<0.05$ V12Rac1 versus TAT using 2-tailed t test. (B) Images show F-actin levels $15 \mathrm{~min}$ after alveolar microinfusions of Rac1 mutants. Scale bars, $50 \mu \mathrm{m}$. Bars are whole-image quantifications of F-actin (rhodamine-phalloidin) (left) at indicated time points and TNFR1 expression (right) 30 min after TNF- $\alpha$ microinfusion in alveoli pretreated with TAT-Rac1 mutants. Mean \pm SEM, $n=5$ lungs for each group. Each dot shows data for a single lung. ${ }^{*} P<0.05$ versus corresponding baseline (dashed line) using ANOVA with Bonferroni correction. (C-E) Immunoblots and densitometry are for lungs given indicated instillations. Tissues were harvested at 4 ( $C$ and $D)$, or 24 (E) hours after instillations. Bands in (C) are immunoblots on lysates that were fractionated as triton-insoluble (F-actin), or -soluble (G-actin), or not fractionated (Actin). Bands in (E) were obtained on immunoprecipitates of His-tagged, exogenous Rac1 mutants. PBS, control; V12, TAT-V12Rac1; N17, TAT-N17Rac1; JP, jasplakinolide. All samples in blots were run simultaneously. Each blot was replicated 4 times (C) or 6 times (D and $\mathbf{E}$ ). Each dot shows data for a single lung. ${ }^{*} P<0.05$ compared with PBS using ANOVA with Bonferroni correction.

conditions (Supplemental Figure 4), the flow cytometry analyses affirm this finding and show further that endothelial uptake did not take place after LPS treatment. Taken together, our findings indicate that LPS decreased epithelial F-actin by a cofilin-mediated mechanism, increasing TNFR1 expression at the epithelial surface, augmenting lung inflammation and, hence, mortality. We conclude that V12Rac1 succeeded in increasing survival in LPS-induced lung injury by both prophylactic and therapeutic strategies.

LPS, an outer coat protein of gram-negative bacteria, causes ALI by activating TLR4-induced proinflammatory signaling in pulmonary alveoli (7). However, gram-negative bacteria such as Pseudomonas aeruginosa (PAO1) may induce ALI through additional mechanisms, as - for example - by production of exotoxin (35). To determine the efficacy of actin enhancement therapy in ALI due to live bacterial infection, we i.n. instilled PA at a dose that causes high mortality. Fifteen hours after instillation, mouse mortality was $100 \%$ (Figure $5 \mathrm{G}$ ). To determine therapeutic efficacy of i.n. TAT-V12Rac1, we administered the construct 4 hours after bacterial instillation. At this time point, lung injury was well developed, as indicated by the extravascular lung water (EVLW), which was 2 times above baseline (Supplemental Figure 8). 


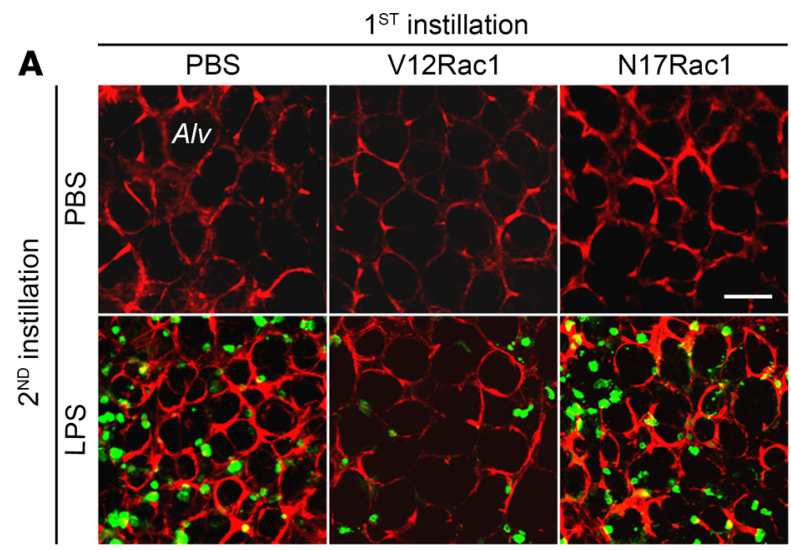

Fluorophores: TNFR1; Ly6G

\section{D}
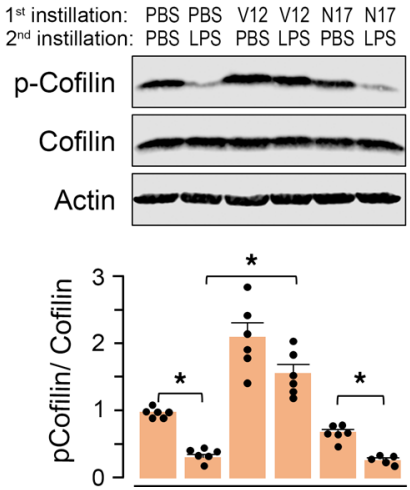

E

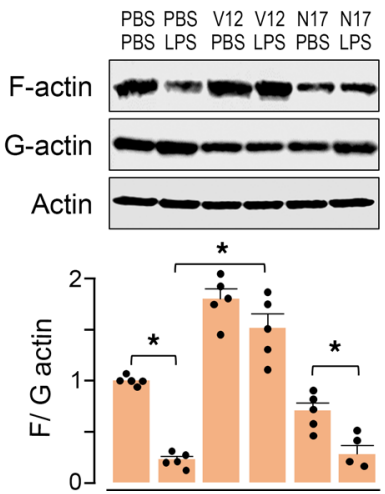

B

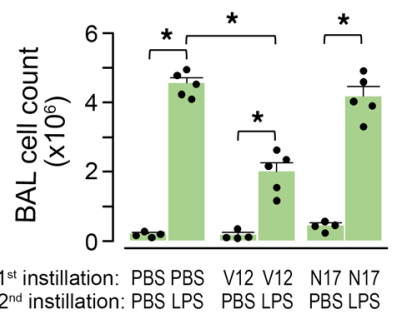

C

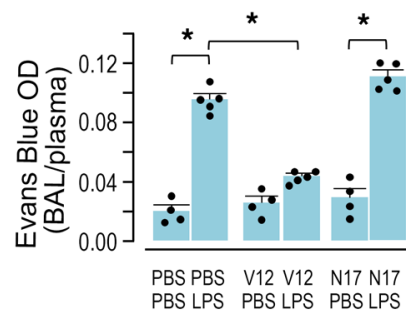

$\mathbf{F}$

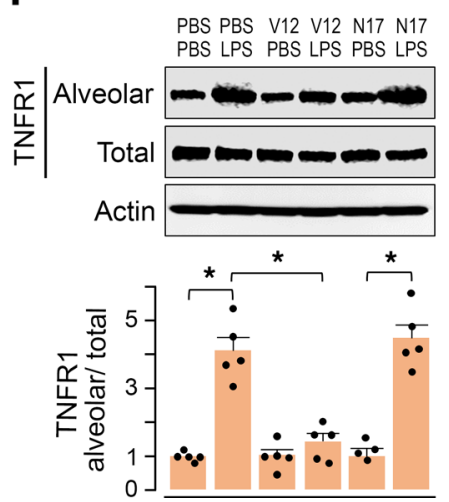

G
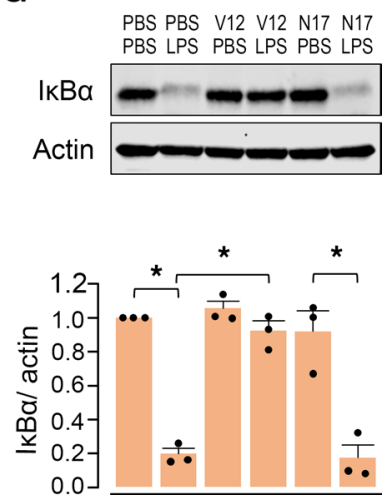

Figure 4. Effects of Rac1 mutants on alveolar F-actin and TNFR1 expression after LPS. Mice were given 2 i.n. instillations each, as indicated. Mice were separately given a first instillation of PBS, TAT-V12Rac1, or N17-Rac1. In each mouse, a second instillation of PBS or LPS (sublethal dose) was given after 30 minutes. Data were obtained 24 hours after LPS. (A) Confocal images show alveolar immunofluorescence of TNFR1 (red) and of neutrophils, detected by Ly6C immunofluorescence (green) after indicated treatments. Abs were microinjected in alveoli. Scale bar: $50 \mu \mathrm{m}$. Alv, alveolus. Replicated in 3 lungs for each group. (B and C) Bars show the total leukocyte count in BAL (B) and alveolar permeability to albumin (C). OD, optical density of Evans blue-bound albumin. $n=4$ mice in PBS treated groups, $n=5$ mice in LPS treated groups. (D-G) Lung immunoblots and densitometry are for indicated proteins for total lung lysates, and for triton-insoluble (F-actin) and -soluble (G-actin) fractions of lung lysates. Alveolar, streptavidin pulldown of epithelium biotinylated in situ. Data are presented as mean \pm SEM. Each dot shows data for a single lung. $n=5-6$ except in $\mathbf{G}$, as indicated by the dots. ${ }^{*} P<0.05$ as indicated using ANOVA with Bonferroni correction.

Despite the severe lung injury, i.n. TAT-V12Rac1 decreased mortality to approximately $20 \%$ at 15 hours. Thus, a postinjury therapeutic strategy with i.n. TAT-V12Rac1 protected against ALI caused by instillation of highly toxic bacteria.

\section{Discussion}

We report here the first definitive evidence to our knowledge that TNFR1 ligation induced loss of the F-actin fence in the alveolar epithelium, causing receptor hyperexpression and alveolar injury. Rac1 delivery to the alveolar epithelium enhanced the fence, blocking the receptor hyperexpression and injury. Of translational significance, the fence enhancement protected survival in LPS- and PA-induced ALI. Together, these findings, to our knowledge, constitute the first evidence that the F-actin fence of the alveolar epithelium is a determinant of lung inflammation and injury.

We propose a sequence of events (Figure 6) in which an inhaled pathogen induces macrophage-derived TNF- $\alpha$ release. TNFR1 ligation on the alveolar epithelium increases epithelial $\mathrm{Ca}^{2+}$, activating the $\mathrm{Cn}$-cofilin pathway to depolymerize F-actin. The actin fence is, thus, disabled, causing a surge of proinflammatory receptor expression. The F-actin depolymerizing agent cytD also caused the receptor surge, indicating that loss of F-actin was necessary and sufficient for the effect and that nonspecific receptor-mediated mechanisms were not required. Importantly, a brief TNF- $\alpha$ exposure induced sustained F-actin decrease in the alveolar epithelium, leading to the receptor surge, which lasted several hours, 
A

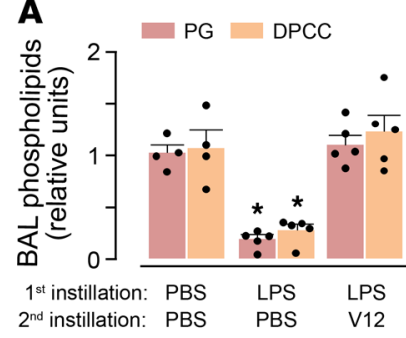

B

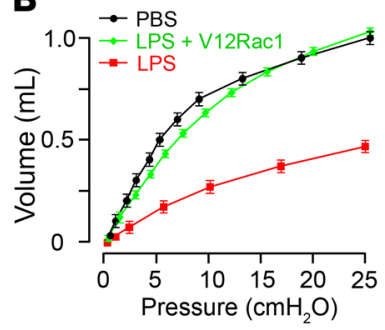

C

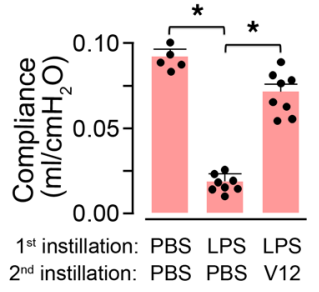

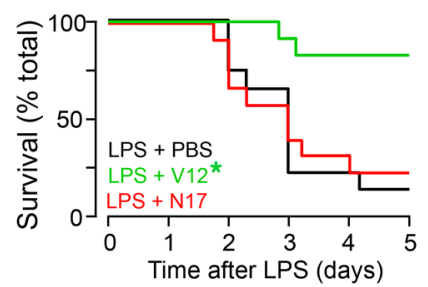

D

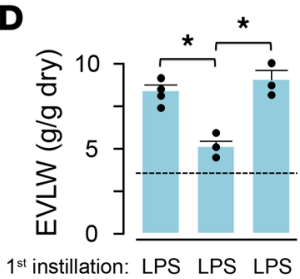

$1^{\text {st }}$ instillation: LPS LPS LPS
$2^{\text {nd }}$ instillation: PBS V12 N17

$48 \mathrm{~h}$
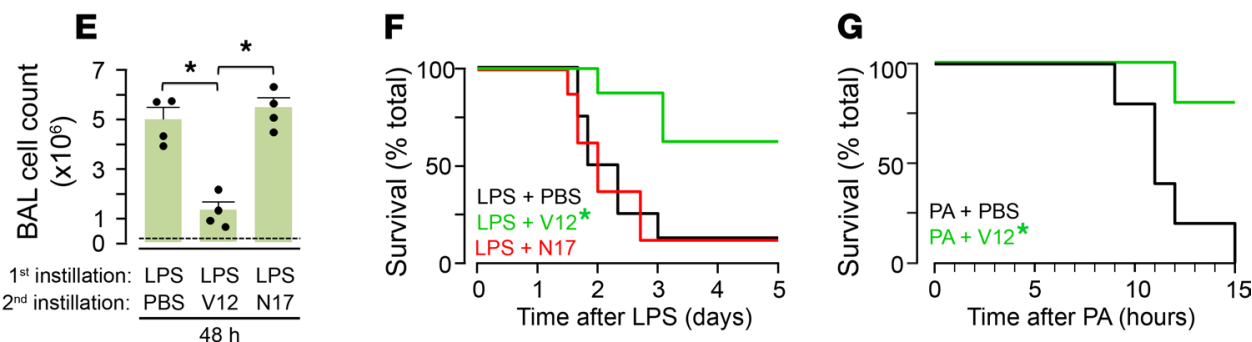

Figure 5. Protective effects of alveolar F-actin enhancement on ALI. (A-F) Mice received 2 i.n. instillations. The first instillation was a lethal dose of LPS. This was followed by the second instillation, which was PBS, or TAT-conjugated proteins. The second instillation was given 4 (A-C) or 24 (D-F) hours after LPS instillation. The data were obtained 72 hours after LPS for $\mathbf{A}$ and $\mathbf{B}$, and at the indicated times for $\mathbf{C}-\mathbf{C}$. (A) Bars show BAL phospholipids levels as indicated. Mean \pm SEM. ${ }^{*} P<0.05$ versus corresponding PBS group using ANOVA with Bonferroni correction. $n=5$, except PBS ( $\left.n=4\right)$. Each dot shows data for a single lung. (B) Bars show lung compliance calculated from the volume-pressure plot. Mean $\pm \mathrm{SEM}$. ${ }^{*} P<0.05$ using ANOVA with Bonferroni correction. $n=8$, except PBS $(n=5)$. Each dot in bar diagram shows data for a single lung. (C) Kaplan-Meier plots for mouse survival. For each group, $n=12$. ${ }^{*} P<0.01$ versus LPS + PBS using log-rank test. ( $D$ and $\mathbf{E}$ ) Data were obtained 48 hours after LPS. Bars show blood-free extravascular lung water (EVLW) (D) and total leukocyte count in BAL (E). Mean $\pm S E M$. ${ }^{*} P<0.001$ using ANOVA with Bonferroni correction. $n=4$, except TATN17Rac1 in $\mathbf{D}(n=3)$. Each dot shows data for a single lung. Baseline values shown as dashed line. (F) Kaplan-Meier plots for mouse survival after LPS. For each group, $n=8$. ${ }^{*} P<0.01$ versus LPS + PBS using log-rank test. (C) Kaplan-Meier plots for mouse survival after instillation of $P$. aeruginosa (PA), followed 4 hours later by TAT-Rac1 proteins. For each group, $n=5$. ${ }^{*} P<0.01$ versus PA + PBS using log-rank test.

possibly corresponding to the time taken for F-actin to return to baseline levels and, thereby, reestablish fence conditions. The prolonged duration of the receptor surge suggests that receptor hyperexpression might be sustained during the inflammatory process if F-actin fence properties are not reestablished. The extent to which this interpretation applies to other proinflammatory receptors expressed on the epithelium (2) requires further investigation.

Actin depolymerization also destabilizes cell-junctional barriers (36), causing increased alveolar permeability to plasma proteins. Repetitions of this sequence of events may perpetuate lung injury, progressively increasing its severity and leading to the high mortality of ARDS. The fact that Rac1 delivery to the alveolar epithelium inhibited this injurious sequence indicates that actin enhancement in the epithelium was sufficient for opposing 2 critical players in the injury response - namely, hyperexpression of proinflammatory receptors and weakening of the epithelial fluid barrier. Since we did not detect exogenous Rac1 in the capillary endothelium, we interpret that V12Rac1's protective effects were predominantly due to epithelial actin enhancement.

The cell-surface expression of the receptor was AT1 restricted, with no detectable AT2 expression. TNFR1 expression on AT1 related inversely to F-actin levels, indicating that the fence was a determinant of the expression even under unstressed conditions at baseline. Cultured, AT2-like A549 cells express TNFR1 (37), although these cells do not entirely reflect properties of AT2 in situ. Interestingly, as we reported (33), and confirm here, F-actin is 3-fold higher in AT2 than AT1, suggesting that high F-actin restricts AT2 expression of cell-surface TNFR1.

Our findings reveal a potentially novel understanding of $\mathrm{Ca}^{2+}$-induced $\mathrm{F}$-actin regulation. An increase of the cytosolic $\mathrm{Ca}^{2+}$ may affect $\mathrm{F}$-actin differently in different functional contexts. In the context of endothelial barrier regulation, $\mathrm{Ca}^{2+}$ increases lead to actin polymerization - hence, stress fiber formation and barrier loss (38-40). By contrast, $\mathrm{Ca}^{2+}$ increases due to $\mathrm{T}$ cell receptor engagement at the immunological synapse induce actin depolymerization, thereby promoting the intensity and duration of $\mathrm{T}$ cell engagement (41). Here, the TNF- $\alpha$-induced $\mathrm{Ca}^{2+}$ transient caused actin depolymerization, as evident in the rapid F-actin decrease. This response - as well as the ensuing TNFR1 surge - was inhibited by blocking the 


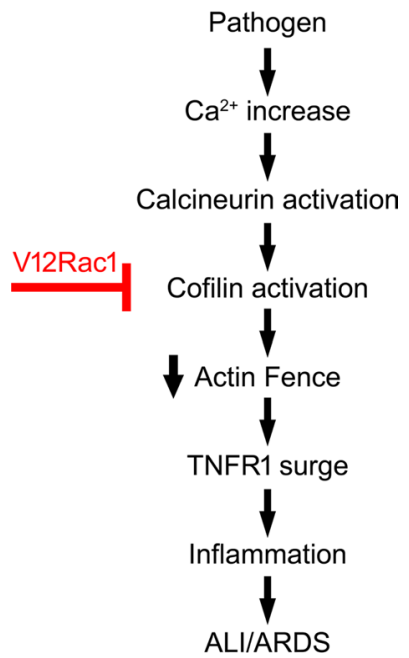

Figure 6. F-actin in acute lung injury. Sequence of events shows signaling mechanisms leading to F-actin-dependent proinflammatory receptor surge during Acute Lung Injury.

$\mathrm{Ca}^{2+}$ transient with calcium chelator BAPTA-AM. Therefore, the cytosolic $\mathrm{Ca}^{2+}$ is a major fence regulatory mechanism that determines TNFR1 display on the alveolar epithelium.

The $\mathrm{Ca}^{2+}$ effect on F-actin was mediated through the Cn-cofilin pathway. Several findings support this interpretation. Thus, the TNF- $\alpha$ effects on F-actin and the surge were absent after alveolar treatment with FK-506, or in $\mathrm{CnA} \beta$-null mice, implicating $\mathrm{Cn}$ in the responses. We interrogated the mechanistic pathway through epithelial expression of constitutively active or inactive mutants of cofilin. The active mutant, which causes $\mathrm{F}$-actin depolymerization, enhanced the TNF- $\alpha$-induced TNFR1 surge. The inactive mutant, which increased F-actin, blocked all TNF- $\alpha$-induced responses, including the surge. These results are further evidence that loss of the actin fence was critical for epithelial hyperexpression of the proinflammatory receptor. Taken together, these findings reveal a sequence of fast-acting signaling events in which a receptor-mediated $\mathrm{Ca}^{2+}$ increase activated calcineurin, leading to cofilin-mediated loss of the F-actin fence and resulting in enhanced proinflammatory receptor display on the alveolar surface.

LPS induced the expected lung inflammation and alveolar injury, as indicated by the presence of albumin leak and significant mortality $(7,34)$. LPS induces secretion of multiple cytokines, including TNF- $\alpha$, while also increasing alveolar epithelial $\mathrm{Ca}^{2+}(7)$. Similar to TNF- $\alpha$, alveolar LPS exposure also dephosphorylated cofilin and decreased F-actin, while increasing TNFR1 expression. However, importantly, all of these LPS effects were sustained for 24 hours. This finding indicates that mortality resulted from prolonged loss of the F-actin fence that caused prolonged TNFR1 hyperexpression, exacerbating alveolar inflammation. We tested this possibility by means of a CPP strategy where the plan was to increase the actin fence through intracellular delivery of V12Rac1 in order to phosphorylate and, therefore, inactivate cofilin.

Our strategy was highly successful. Whether administered by alveolar microinjection or by the i.n. route, the TAT-V12Rac1 construct was rapidly internalized by the alveolar epithelium. The $\mathrm{pH}$-sensitive, noncovalently conjugated construct hydrolyzed intracellularly, enabling TAT diffusion out of the cell. Therefore, although the protein cargo was successfully taken up by the epithelium, TAT — which contains a nuclear-localizing sequence (42) and may therefore induce unwanted transcriptional effects - was eliminated. We consider this TAT elimination from the alveolar epithelium a strength of our CPP strategy, since such an elimination would abrogate the likelihood of transcription-induced long-term toxicity due to TAT. Although reports indicate that extracellular TAT is rapidly removed from the lung $(43,44)$, further studies are required to understand mechanisms underlying the removal process.

The instilled TAT-V12Rac1 construct was not detectable in the capillary endothelium before or after induction of alveolar inflammation. Thus, once dissociated from TAT, nonconjugated V12Rac1 protein remained confined to the epithelial cytosol and did not enter endothelial cells. We affirmed this result by assaying the membrane permeabilizing effects of saponin on cell fluorescence. Given by alveolar injection, saponin removed epithelial but not endothelial fluorescence (Supplemental Figure 4), indicating that the construct was localized only to the alveolar compartment. This conclusion is further supported by our flow 
cytometry results showing that - even after LPS treatment, a condition that increased alveolar permeability - there was no endothelial uptake of V12Rac1 (Supplemental Figure 7C). Therefore, TAT-V12Rac1 did not modify endothelial F-actin. We consider it unlikely that it directly affected endothelial barrier determinants, such as focal adhesions and junctional proteins (45-47).

Following delivery of the CPP, we could detect V12Rac1 in the epithelium for at least 24 hours, during which epithelial F-actin also remained elevated and the TNFR1 hyperexpression was abrogated. These findings show that constitutively active Racl increases F-actin in vivo and that the induced F-actin remains stable for sufficient durations to be therapeutically effective. Importantly, our findings indicate that pretreatment with i.n. TAT-V12Rac1 markedly reduced mortality due to LPS. We point out, however, that while our studies support a protective effect of the actin fence in the context of TNFR1 expression, the extent to which actin fence strengthening modifies the effects of other ALI-relevant receptors, such as the proinflammatory IL-1 $\beta$ receptor or the antiinflammatory TNFR2 (48), requires further study. Nevertheless, our findings indicate that a pretreatment strategy with V12Rac1 might be a feasible prophylactic option against ALI.

To determine the therapeutic efficacy of TAT-V12Rac1 as a curative agent, we instilled the construct under conditions of established pathology, namely 4 or 24 hours after instilling a lethal LPS dose that caused major alveolar hyperpermeability and inflammation. These post-LPS V12Rac1 instillations were also protective against mortality. An important conclusion is that fence enhancement in the alveolar epithelium mitigates ALI well into the progressive phase of the disease. However, since the mortality protection was less for the delayed intervention group, fence enhancement therapy might be more effective in early rather than late stages of ALI.

We also considered that infection by live bacteria may induce injury mechanisms that are more extensive than those due to LPS alone. Accordingly, we determined the effects of the construct in the presence of lung infection by a highly LD of PA, an organism that is a major cause of ALI and ARDS (49). In WT mice, this dose of PA induced catastrophic mortality in 15 hours. However, when we gave TAT-V12Rac1 after inducing infection, the mortality was markedly abrogated. These findings add to the translational significance of our study, which reveals the therapeutic strength of V12Rac1 in the setting of ongoing lung inflammation.

$\mathrm{Ab}$ inhibition of TNFR1 has been advanced as a possible therapeutic strategy for ALI $(50,51)$. However, Ab therapy was protective for ALI not associated with mortality $(50,51)$. Therefore, these reported findings are not directly comparable with the present mortality-causing inflammatory responses that could be encountered in clinical ARDS. In severe inflammation, cell-directed therapy as we propose may be more effective.

Since Rac1 plays a role in multiple cellular processes (52-54), we considered whether its sustained epithelial presence affected alveolar function. We approached this issue by quantifying lung surfactant secretion and lung compliance, metrices that report adequacy of overall alveolar homeostasis. Maintenance of surfactant secretion reflects adequacy of several epithelial regulatory parameters, such as cytosolic $\mathrm{Ca}^{2+}$ regulation (55). The fact that V12Rac1 protected these critical functional responses despite ALI indicates that V12Rac1 treatment not only did not interfere with major aspects of epithelial function, but that the treatment reinstated alveolar homeostasis in ALI.

Although our study indicates that there were no overall negative consequences attributable to V12Rac1, to support clinical application, further data are required to clarify issues including the dynamics of elimination of the internalized V12Rac1, as well as the protective efficacy in large-animal models of ALI. Since pharmacologic therapy for ALI remains elusive, and since the therapeutic efficacy of cellular delivery of protein constructs has not been previously evaluated, we conclude that our potentially novel V12Rac1 construct may have potential as therapeutic strategy for ALI, as well as for severe inflammatory diseases in other organs.

\section{Methods}

Reagents. Fluorophores calcein AM (5-10 $\mu \mathrm{M})$, Calcein Red-Orange AM (5 $\mu \mathrm{M})$, LTR (100nM), Fluo-4 (10 $\mu \mathrm{M})$, Alexa-488-phalloidin, and Rhodamine-phalloidin were purchased from Invitrogen. Human recombinant TNF- $\alpha$ (10-100 ng/mL, BD Biosciences), and BAPTA-AM (100 $\mu \mathrm{M}$, Invitrogen) were used, as well as FK506 $(100 \mu \mathrm{M})$, LPS $(1-10 \mathrm{mg} / \mathrm{kg})$, cytocholasin D $(100 \mathrm{nM})$, jasplakinolide $(100 \mathrm{nM})$, latrunculin B (50 nM), TB (0.01\%), DTT (1 mM), Turk's solution, and TB, which were purchased from Sigma-Aldrich. Protein A/G-agarose beads were purchased from Santa Cruz Biotechnolgoy Inc. Saponin was purchased from Calbiochem. EZ-Link $N$-hydroxysuccinimide-SS-biotin (catalog 21331) and streptavidin-Sepharose beads (catalog 20357) were purchased from Thermo Fisher Scientific. 
Abs. mAb MCA2350 against the TNFR1 extracellular epitope was purchased (AbD Serotec). Abs were fluorescently labeled with Alexa Fluor 633 using standard protocols. Ab against GFP (catalog sc-9996) and $\mathrm{I} \kappa \mathrm{B} \alpha \mathrm{Ab}$ (catalog sc-371-G) were from Santa Cruz Biotechnology Inc. For immunoblotting experiments, we purchased Abs against p-Cofilin (catalog 3313) and Cofilin (catalog 5175) from Cell Signaling Technology. Abs against TNFR1 (catalog sc-8436), His-probe (catalog sc-8036), and Rac1 (catalog sc-6084) were from Santa Cruz Biotechnology Inc. Anti-actin Ab (catalog 2066) was purchased from MilliporeSigma. Fluorescence-tagged Abs against mouse CD45 (catalog 103126) were from BioLegend, and anti-mouse Ly6G (catalog 11-9668), CD31 (catalog 12-0311), and T1 $\alpha$ (catalog 25-5381) were from eBiosciences.

Animals. Pathogen-free, adult male WT Swiss Webster mice (6-10 weeks old) were purchased from Taconic Biosciences. CnA $\beta$-null mice were provided by Jeffery D. Molkentin (Divisions of Molecular Cardiovascular Biology Children's Hospital Medical Center, Cincinnati, Ohio, USA). Age-, sex-, and strainmatched WT mice were purchased from the Jackson Laboratory. For mouse anesthesia for i.n. instillations and surgical procedures, we gave inhaled isoflurane (3\%) and i.p. injections of ketamine and xylazine (100 $\mathrm{mg} / \mathrm{kg}$ and $5 \mathrm{mg} / \mathrm{kg}$, respectively).

Isolated, blood-perfused lungs. Lungs were prepared according to our reported method $(7,34,56)$. Briefly, we excised intact lungs from anesthetized mice. Lungs were inflated through a tracheal cannula with a gas mixture $\left(30 \% \mathrm{O}_{2}, 6 \% \mathrm{CO}_{2}\right.$, balance $\left.\mathrm{N}_{2}\right)$ and were continuously pump-perfused with autologous blood (final hematocrit, $10 \%$ ) through cannulas in the pulmonary artery and left atrium at a constant flow rate of $0.4-0.5 \mathrm{~mL} / \mathrm{minute}$ at $37^{\circ} \mathrm{C}$. Blood was diluted in HEPES buffer $\left(150 \mathrm{mM} \mathrm{Na}^{+}, 5 \mathrm{mM} \mathrm{K}^{+}, 1.0 \mathrm{mM} \mathrm{Ca}^{2+}, 1 \mathrm{mM} \mathrm{Mg}^{2+}\right.$, and $20 \mathrm{mM}$ HEPES at pH 7.4) supplemented with $4 \%$ dextran (70 kDa; TCI America) and 1\% FBS (HyClone, Thermo Fisher Scientific) at osmolarity 295 mosM (Fiske Micro-Osmometer, Fiske Associates). Airway, pulmonary artery, and left atrial pressures were held constant at 5,10 , and $3 \mathrm{~cm} \mathrm{H}_{2} \mathrm{O}$, respectively, during microscopy.

Alveolar microinjection and in situ immunofluorescence. We used isolated blood-perfused mouse lungs for imaging experiments. To load the alveolar epithelium with fluorophores, reagents, solutions, and Abs, we micropunctured single alveoli with hand-beveled glass micropipettes (opening diameter 5-8 $\mu \mathrm{m}$ ) under bright-field microscopy and microinstilled alveoli with solutions. Instilled solutions spread from the micropunctured alveolus to at least 15 neighboring alveoli within lung acini $(57,58)$. To carry out live immunofluorescence, alveolar $\mathrm{Ab}$ infusions were followed by washout with PBS. To assess fluorophore internalization, microinfusion of fluorophore-conjugated agents was followed by alveolar infusion of the membrane-impermeable fluorescence quencher TB (0.01\% solution, 10 minutes) (9).

To carry out in situ rhodamine-phalloidin staining and anti-GFP immunofluorescence, alveoli were fixed by continuous microinfusion of $4 \%$ paraformaldehyde (15 minutes) and permeabilized by infusion of $0.1 \%$ Triton X-100 (5 minutes). Then, alveoli were microinfused with fluorescence-conjugated (Alexa Fluor 488 or rhodamine) phalloidin or fluorescence-tagged antibody for 10 minutes. For Ab staining, we blocked the tissue for 20 minutes with 1\% FBS and then microinfused primary Ab (30 minutes) and secondary Ab (30 minutes) in $1 \%$ FBS containing HEPES buffer. Unbound agents were removed by a 10-minute washout with HEPES buffer containing $1 \%$ FBS and $0.01 \%$ Tween 20 .

Live alveolar imaging. We imaged alveoli of isolated, blood-perfused lungs, held at constant inflation, by laser scanning confocal microscopy (LSM 510; Carl Zeiss Microscopy) using a $\times 40$ water immersion objective (numerical aperture 0.80, Achroplan; Carl Zeiss Microscopy) per our reported protocol (7, 34, 56). During concurrent application of multiple fluorophores, we confirmed the absence of bleed-through between different fluorescence emission channels. As indicated in the figure legends, fluorescence was quantified across a gray level range of 10-255 for specified regions of interest (ROIs), or for the entire image (MetaMorph 7.8, Universal Imaging). To eliminate background fluorescence, the fluorescence gain was set to give images with zero gray levels in the absence of added fluorophore. Laser power, detector gain, and optical thickness were held constant across experiments for each fluorophore. In case of an excessive fluorescence response, we reduced gain to prevent fluorescence saturation and then corrected gray levels against the linear relationship between gray levels and gain that we determined for each fluorophore.

We imaged TNFR1 expression as immunofluorescence of an ectodomain-specific fluorescent $\mathrm{Ab}$ (mAb MCA2350; AbD Serotec) (9), which we gave by alveolar micropuncture. To image F-actin expression, we detected fluorescence of a transfected F-actin probe (Lifeact-RFP; gift of Roland Wedlich-Soldner, Max Planck Institute, Martinsried, Germany) in live tissue $(33,59)$ or in fixed and permeabilized tissue by rhodamine-phalloidin fluorescence. For $\mathrm{Ca}^{2+}$ imaging, we loaded the alveolar epithelium with the $\mathrm{Ca}^{2+}$ probe, fluo-4 (Invitrogen). $\mathrm{Ca}^{2+}$ and Lifeact images were acquired at 1 image/10 seconds. 
In vivo transfection. Lifeact-RFP construct was a gift from Roland Wedlich-Soldner (Max Planck Institute). Plasmids for WT cofilin ( $p \mathrm{WT})$, an activated mutant ( $p \mathrm{~S} 3 \mathrm{~A})$, and an inactive mutant $(p \mathrm{~S} 3 \mathrm{E})$ were provided by Stuart S. Martin (Department of Physiology, University of Maryland School of Medicine, Baltimore, Maryland, USA) (26). Using our established methods (7, 34, 56), we complexed plasmid DNA (75 $\mu \mathrm{g})$ with freshly extruded unilamellar liposomes $(20 \mu \mathrm{g} / \mu \mathrm{L} ; 100 \mathrm{~nm}$ pore size; DOTAP, Avanti Lipids) in sterile Opti-MEM (Invitrogen). We administered a plasmid DNA-liposomes mix by i.n. instillation in anesthetized mice. Imaging experiments were carried out 48 hours after transfection.

TAT-Rac1 conjugation. We cloned cDNA of V12Rac1 and N17Rac1 (gift from P. Jurdic; Laboratoire de Biologie Moléculaire et Cellulaire, Ecole Normale Supérieure de Lyon, Lyon, France) into a His transfer vector. Then, to make recombinant virus, we cotransfected Sf9 cells with the cloned cDNA and viral DNA (BaculoGold). We infected Sf9 cells with the recombinant virus to express and purify the His-tagged proteins using a nickel-nitrilotriacetic acid (Ni-NTA, Qiagen) column. For noncovalent, $\mathrm{pH}$-dependent conjugation of TAT peptide to the Rac1 proteins, we synthesized nitrilotriacetic acid (NTA) to the N-terminus of TAT (amino acids 48-60, CHI Scientific). Then, we combined equimolar amounts of NTA-TAT (500 $\mu \mathrm{M}$ in $20 \mu \mathrm{L}$ sterile, calcium-free, magnesium-free PBS) and copper sulfate ( $500 \mu \mathrm{M}$ in $20 \mu \mathrm{L}$ deionized water) for 30 minutes at room temperature to obtain copper-containing NTA-TAT. Purified His-Rac1 (V12Rac1 or N17Rac1) was dissolved in dipositive cation free PBS $(20 \mu \mathrm{L})$ and then added to the copper-conjugated NTA-TAT (NTA-TAT-Cu) mixture. The reaction mixture was left at room temperature for 90 minutes. In this final mixture, component volumes were adjusted to establish concentrations of NTA-TAT at $50 \mu \mathrm{M}$ and of His-Rac1 at $500 \mu \mathrm{g} / \mathrm{kg}$.

$A L I$. Lung injury was induced by i.n. instillation of LPS in sterile PBS or of live Pseudomonas aeruginosa (PAO1). LPS concentration was $1 \mathrm{mg} / \mathrm{kg}$ (non-LD) for ALI experiments and $10 \mathrm{mg} / \mathrm{kg}$ (LD) for the survival experiments $(7,33)$. PAO1 was given at a concentration of $2.5 \times 10^{7} \mathrm{CFU}$ in $50 \mu \mathrm{L}$ of PBS. TATRac1V12 or TAT-Rac1 N17 (0.5 mg/kg in $40 \mu \mathrm{L}$ PBS) were given by i.n. instillation 30 minutes before or 4 hours after the induction of injury. For control, we instilled an equal volume of sterile PBS. Mice were anesthetized during the instillations.

Bacterial preparation. Pseudomonas aeruginosa (PAO1) was grown on Luria-Bertani (LB; MP Biomedicals) agar at $37^{\circ} \mathrm{C}$. We inoculated $\mathrm{LB}$ broth with a single colony and then grew the bacteria overnight in a shaking incubator at $37^{\circ} \mathrm{C}$ and $250 \mathrm{rpm}$ (Innova42, New Brunswick Scientific). We added $100 \mu \mathrm{L}$ of the overnight broth culture to $10 \mathrm{~mL}$ fresh LB broth and propagated the bacteria in a shaking incubator to OD of 0.5 at $600 \mathrm{~nm}$ (SPECTRAmax Plus, Molecular Devices). For infection, we centrifuged (500 $g, 5$ minutes, room temperature) and resuspended $1 \mathrm{~mL}$ of culture $\left(\mathrm{OD}_{600 \mathrm{~nm}}=0.5\right)$ in $1 \mathrm{~mL}$ of PBS; we then administered $50 \mu \mathrm{L}$ of bacterial suspension to deliver $2.5 \times 10^{7} \mathrm{CFU}$ per mouse.

Analysis of BAL fluid. The lungs of anesthetized mice were lavage 5 times with ice-cold PBS without calcium and magnesium ( $1 \mathrm{~mL}$ each time) through a tracheal cannula. Collected BAL fluids were centrifuged for 10 minutes at $350 \mathrm{~g}$ and $4^{\circ} \mathrm{C}$. For cell counts, pellets were resuspended in $1 \mathrm{~mL}$ of PBS and stained with Turk's solution. Cell counts were determined using a hemocytometer (Hausser Scientific). The supernatant was analyzed for protein concentration. For phospholipid analyses, supernatants were centrifuged $\left(27,000 \mathrm{~g}, 60\right.$ minutes, $\left.4^{\circ} \mathrm{C}\right)$ to obtain a pellet containing the large aggregate (LA) fraction of alveolar surfactant (60-62). The LA fraction was resuspended in sterile PBS, and its phospholipid composition was analyzed by thin-layer chromatography (TLC) using standard procedures following lipid extraction from the LA fraction $(60,61)$. Briefly, a 2:1 (v/v) chloroform/methanol mixture was added to resuspended LA; then, the lipid rich chloroform phase was recovered after vortexing and centrifugation. The chloroform was evaporated to dryness under $\mathrm{N}_{2}$. The lipid extract was reconstituted in $50 \mu \mathrm{L}$ acetonitrile and applied to a TLC plate. Lipids were separated employing a mixture of 65:25:4 (v/v/v) chloroform/methanol/water as a mobile phase followed by lipid visualization in an iodine chamber.

Evaluation of $A L I$. We followed reported protocols for quantifications of alveolar permeability (34) and EVLW $(56,63)$. Briefly, alveolar permeability was quantified as the BAL/plasma ratio of Evans bluealbumin (EB-albumin) concentrations, 4 hours after i.v. EB-albumin. EVLW was the wet/dry ratio of the lung homogenate, corrected for blood water content. To quantify lung compliance (CL), mouse lungs were inflated to $25 \mathrm{~cm} \mathrm{H}_{2} \mathrm{O}$ airway pressure $(\mathrm{P})$. Then, decreases in $\mathrm{P}$ were recorded for step decreases in lung volume (V). CL was calculated as the slope of the linear regression of $\mathrm{V}$ against $\mathrm{P}$ for $\mathrm{P}=0-10 \mathrm{cmH}_{2} \mathrm{O}$. Data for each lung are means of 2 replicated V-P plots. 
Survival assessment. Animals were randomly divided for the experimental groups. Anesthetized mice were treated with i.n. instillation of TAT-Rac1 conjugates $(0.5 \mathrm{mg} / \mathrm{kg}) 30$ minutes before or 4 hours after i.n. instillation of LPS at a LD (10 mg/kg). The mice were scored at frequent intervals following LPS administration, in agreement with the approved Animal Care Protocol. At each assessment, the animals were scored by a blinded investigator applying a scoring system that includes measuring of body weight and evaluation of respiration, activity, and grooming.

Lung cell isolation and flow cytometry. To isolate lung cells, we buffer perfused lungs through vascular cannulas to clear blood; we then minced and passed the tissue through $40 \mu \mathrm{m}$ cell strainers (BD Biosciences) to obtained single cell suspension. For flow cytometry, we surface stained the cells by incubating the suspension with fluorophore-conjugated $\mathrm{Abs}\left(15\right.$ minutes, $\left.4^{\circ} \mathrm{C}\right)$. Alveolar epithelial cells were identified as CD45-CD31- $11 \alpha^{+}$and endothelial cells were identified as $\mathrm{CD} 45^{-} \mathrm{CD} 31^{+} \mathrm{T} 1 \alpha^{-}$. We analyzed cells by flow cytometry (LSR II, BD Biosciences) and using standard software (FlowJo, Tree Star Inc.).

Immunoprecipitation and Western blot analysis. Lungs were cleared from BAL leukocytes and blood by repeated lavage via tracheal cannula and vascular perfusion, respectively, with $10 \mathrm{~mL}$ of ice cold PBS. Freshly dissected lungs were homogenized (Tissue Tearor; Biospec Products) in Pierce RIPA buffer (89901, Thermo Fisher Scientific) containing Halt protease and phosphatase inhibitor cocktail (78442, Thermo Scientific). The lysates were cleared by centrifugation for 20 minutes at $16,000 g\left(4^{\circ} \mathrm{C}\right)$. Protein concentrations were determined using a commercial kit (Pierce BCA Protein Assay Kit; Thermo Fisher Scientific), BSA standards ( $2 \mathrm{mg} / \mathrm{mL}$ in $0.9 \%$ saline; Thermo Fisher Scientific), and a plate reader (SPECTRAmax Plus; Molecular Devices). Samples containing equal amounts (75-100 $\mu \mathrm{g}$ ) of proteins were resuspended in Laemmli sample buffer, boiled for 5 minutes, separated by SDS-PAGE, and electrotransferred onto nitrocellulose membrane (1620115, Bio-Rad) overnight at $4^{\circ} \mathrm{C}$.

Exogenous Rac1 mutant proteins were immunoprecipitated from equal amounts of whole lung lysates $(1 \mathrm{mg})$ with anti-His $\mathrm{Ab}(1 \mu \mathrm{g})$ overnight at $4^{\circ} \mathrm{C}$, and protein A/G PLUS agarose beads $(20 \mu \mathrm{L})$ were added to the samples and incubated for an additional 4 hours at $4^{\circ} \mathrm{C}$. The beads were washed 5 times with lysis buffer, resuspended in $2 \times$ Laemmli sample buffer, and analyzed by SDS-PAGE and Western blotting. We used primary and secondary Abs following the manufacturer's instructions. We imaged the blots using a Kodak molecular imaging station (IS4000MM) and Odyssey Fc Imaging System (LI-COR Biosciences). Quantification of protein levels was performed by densitometry scanning with ImageJ (NIH). Values were normalized to the PBS lane from the same experimental set.

Biotinylation of alveolar surface protein expression. The lungs of anesthetized mice were lavaged $3 \times$ with ice-cold PBS to remove leucocytes from the airspace. Alveolar surface proteins were labeled for 20 minutes using $1 \mathrm{mg} / \mathrm{mL}$ EZ-Link $N$-hydroxysuccinimide-SS-biotin instilled through a tracheal cannula, followed by repeated lavage with PBS containing $50 \mathrm{mM}$ glycine to quench unbound biotin. Lung tissue were lysed in modified RIPA buffer (50 mM Tris- $\mathrm{HCl}$ [pH 8], $150 \mathrm{mM} \mathrm{NaCl}, 1 \% \mathrm{NP}-40,1 \%$ sodium deoxycholate, and protease inhibitors). Biotinylated proteins were pulled down with streptavidin beads overnight at $4^{\circ} \mathrm{C}$ on a rocking platform from $500 \mu \mathrm{g}$ lung lysates and analyzed by SDS-PAGE and Western blotting.

Lung fractioning for G-actin and F-actin. A 2-step solubilization was carried out by our reported method (36). Briefly, lung tissue cleared from blood and BAL leukocytes were homogenized and solubilized in 400 $\mu \mathrm{L}$ of mild lysis buffer (50 mM NaCl, $10 \mathrm{mM}$ PIPES [pH 6.8], $3 \mathrm{mM} \mathrm{MgCl} 2,0.05 \%$ Triton X-100, $300 \mathrm{mM}$ sucrose) for 20 minutes at $4^{\circ} \mathrm{C}$ on a rocking platform. The whole lung lysates were clarified by centrifugation at $10,000 \mathrm{~g}$ for 10 minutes $\left(4^{\circ} \mathrm{C}\right)$. The supernatant, Triton-X 100 soluble fraction, was collected and used to determine G-actin. The resulting pellet was resuspended in $200 \mu \mathrm{L}$ of stronger solubilizing buffer $(15 \mathrm{mM}$ Tris [pH 7.5], $5 \mathrm{mM}$ EDTA, $2.5 \mathrm{mM}$ EGTA, 1\% SDS) and incubated at $95^{\circ} \mathrm{C}$ for 10 minutes to dissolve the pellet; then, volume was adjusted to $400 \mu \mathrm{L}$ with RIPA buffer. The resulting lysates were clarified by centrifugation at $16,000 \mathrm{~g}$ for 10 minutes $\left(4^{\circ} \mathrm{C}\right)$. Collected supernatant, Triton-X 100 insoluble fraction, was used to determine F-actin. Extracted proteins $(15 \mu \mathrm{g})$ were subjected to SDS-PAGE and Western blotting analysis.

Statistics. Group numbers were designed to enable detection of statistically significant differences with a power of $85 \%$. For imaging experiments carried out with a paired protocol, baseline and test conditions were obtained in the same alveoli, and at least 5 determinations were obtained for each lung. These determinations were averaged to obtain a mean for each condition in each lung. There was no statistically significant within-lung variability of effect size. The means for each lung were pooled for the group to obtain mean \pm SEM, where $n$ represents the number of lungs. The per-lung means are shown in the bar diagrams. Group means were compared by 1-way ANOVA with Bonferroni correction. Data for the same alveolar 
segment were compared by the paired 2-tailed $t$ test. Survival rates were analyzed by the Kaplan-Meyer log rank test. We accepted significance at $P<0.05$.

Study approval. All animal procedures were reviewed and approved by the IACUC at Columbia University Medical Center.

\section{Author contributions}

GAG designed, carried out, and analyzed all experiments; SRD and GJ carried out the recombinant protein purification and conjugation; MNI contributed to the imaging experiments and survival studies; KW contributed to calcium imaging experiments; IOS contributed to the TLC experiments; LL contributed to the EVLW experiments; SB contributed to the plan; and JB designed the overall project and wrote the final manuscript. All authors contributed to the writing.

\section{Acknowledgments}

M. Wei assisted with Rac1 protein expression. GAG, SRD, MNI, GJ, and JB were supported by grants DoD-PR150672, R01 HL36024, and R01 HL57556; IOS was supported by grants R01 DK068437, R01 DK101251, and R01 DK122071. The Flow Cytometry facility was supported by NIH grants S10RR027050, S10OD020056, 5P30DK063608, and P30CA013696.

Address correspondence to: Jahar Bhattacharya, Department of Medicine, 630 W. 168th Street, Room BB 8-812, New York, New York 10032, USA. Phone: 212.305.7093; Email: jb39@cumc.columbia.edu.

1. Bellani G, et al. Epidemiology, patterns of care, and mortality for patients with acute respiratory distress syndrome in intensive care units in 50 countries. JAMA. 2016;315(8):788-800.

2. Liang J, et al. Hyaluronan and TLR4 promote surfactant-protein-C-positive alveolar progenitor cell renewal and prevent severe pulmonary fibrosis in mice. Nat Med. 2016;22(11):1285-1293.

3. Thorley AJ, et al. Differential regulation of cytokine release and leukocyte migration by lipopolysaccharide-stimulated primary human lung alveolar type II epithelial cells and macrophages. J Immunol. 2007;178(1):463-473.

4. Patel BV, et al. TNF-induced death signaling triggers alveolar epithelial dysfunction in acute lung injury. J Immunol. 2013;190(8):4274-4282.

5. Kuebler WM, et al. A novel signaling mechanism between gas and blood compartments of the lung. J Clin Invest. 2000;105(7):905-913.

6. Pease JE, Sabroe I. The role of interleukin-8 and its receptors in inflammatory lung disease: implications for therapy. Am J Respir Med. 2002;1(1):19-25.

7. Westphalen K, et al. Sessile alveolar macrophages communicate with alveolar epithelium to modulate immunity. Nature. 2014;506(7489):503-506.

8. Zhang P, et al. Innate immunity and pulmonary host defense. Immunol Rev. 2000;173:39-51.

9. Rowlands DJ, et al. Activation of TNFR1 ectodomain shedding by mitochondrial Ca2+ determines the severity of inflammation in mouse lung microvessels. J Clin Invest. 2011;121(5):1986-1999.

10. Deng M, et al. Shedding of the tumor necrosis factor (TNF) receptor from the surface of hepatocytes during sepsis limits inflammation through cGMP signaling. Sci Signal. 2015;8(361):ra11.

11. Wang J, et al. Histamine antagonizes tumor necrosis factor (TNF) signaling by stimulating TNF receptor shedding from the cell surface and Golgi storage pool. J Biol Chem. 2003;278(24):21751-21760.

12. Morton PE, et al. TNFR1 membrane reorganization promotes distinct modes of TNF $\alpha$ signaling. Sci Signal. 2019;12(592):eaaw2418.

13. Gomez MI, et al. Staphylococcus aureus protein A activates TACE through EGFR-dependent signaling. EMBO J. 2007;26(3):701-709

14. Neznanov N, et al. Poliovirus protein $3 \mathrm{~A}$ inhibits tumor necrosis factor (TNF)-induced apoptosis by eliminating the TNF receptor from the cell surface. $J$ Virol. 2001;75(21):10409-10420.

15. Storey $\mathrm{H}$, et al. The p55 tumour necrosis factor receptor TNFR1 contains a trans-Golgi network localization signal in the C-terminal region of its cytoplasmic tail. Biochem J. 2002;366(Pt 1):15-22.

16. Bradley JR, et al. Disparate localization of 55-kd and 75-kd tumor necrosis factor receptors in human endothelial cells. Am J Pathol. 1995;146(1):27-32.

17. Gu J, et al. ADF/cofilin-mediated actin dynamics regulate AMPA receptor trafficking during synaptic plasticity. Nat Neurosci. 2010;13(10):1208-1215

18. Wollman R, Meyer T. Coordinated oscillations in cortical actin and Ca2+ correlate with cycles of vesicle secretion. Nat Cell Biol. 2012;14(12):1261-1269.

19. Casella JF, et al. Cytochalasin D inhibits actin polymerization and induces depolymerization of actin filaments formed during platelet shape change. Nature. 1981;293(5830):302-305

20. Rusnak F, Mertz P. Calcineurin: form and function. Physiol Rev. 2000;80(4):1483-1521.

21. Lara-Pezzi E, et al. A naturally occurring calcineurin variant inhibits FoxO activity and enhances skeletal muscle regeneration. J Cell Biol. 2007;179(6):1205-1218

22. Bueno OF, et al. Impaired cardiac hypertrophic response in Calcineurin Abeta -deficient mice. Proc Natl Acad Sci U S A. 
2002;99(7):4586-4591.

23. Descazeaud V, et al. Calcineurin regulation of cytoskeleton organization: a new paradigm to analyse the effects of calcineurin inhibitors on the kidney. J Cell Mol Med. 2012;16(2):218-227.

24. Zhang XF, et al. Calcineurin-dependent cofilin activation and increased retrograde actin flow drive 5-HT-dependent neurite outgrowth in Aplysia bag cell neurons. Mol Biol Cell. 2012;23(24):4833-4848.

25. Moriyama K, et al. Phosphorylation of Ser-3 of cofilin regulates its essential function on actin. Genes Cells. 1996;1(1):73-86.

26. Vitolo MI, et al. Loss of PTEN induces microtentacles through PI3K-independent activation of cofilin. Oncogene. 2013;32(17):2200-2210.

27. Nagumo Y, et al. Cofilin mediates tight-junction opening by redistributing actin and tight-junction proteins. Biochem Biophys Res Commun. 2008;377(3):921-925.

28. Yang N, et al. Cofilin phosphorylation by LIM-kinase 1 and its role in Rac-mediated actin reorganization. Nature. 1998;393(6687):809-812.

29. Edwards DC, et al. Activation of LIM-kinase by Pak1 couples Rac/Cdc42 GTPase signalling to actin cytoskeletal dynamics Nat Cell Biol. 1999;1(5):253-259.

30. Zhang L, et al. Regulation of cofilin phosphorylation and asymmetry in collective cell migration during morphogenesis. Development. 2011;138(3):455-464.

31. Delorme V, et al. Cofilin activity downstream of Pak1 regulates cell protrusion efficiency by organizing lamellipodium and lamella actin networks. Dev Cell. 2007;13(5):646-662.

32. Quadri SK, Bhattacharjee M, Parthasarathi K, Tanita T, Bhattacharya J. Endothelial barrier strengthening by activation of focal adhesion kinase. J Biol Chem. 2003;278(15):13342-13349.

33. Islam MN, et al. F-actin scaffold stabilizes lamellar bodies during surfactant secretion. Am J Physiol Lung Cell Mol Physiol. 2014;306(1):L50-L57.

34. Islam MN, et al. Mitochondrial transfer from bone-marrow-derived stromal cells to pulmonary alveoli protects against acute lung injury. Nat Med. 2012;18(5):759-765.

35. Michalska M, Wolf P. Pseudomonas exotoxin a: optimized by evolution for effective killing. Front Microbiol. 2015;6:963.

36. Quadri SK, et al. Cadherin selectivity filter regulates endothelial sieving properties. Nat Commun. 2012;3:1099.

37. Parsons PE, et al. Elevated plasma levels of soluble TNF receptors are associated with morbidity and mortality in patients with acute lung injury. Am J Physiol Lung Cell Mol Physiol. 2005;288(3):L426-L431.

38. Gudermann T, Steinritz D. STIMulating stress fibers in endothelial cells. Sci Signal. 2013;6(267):pe8.

39. Shinde AV, et al. STIM1 controls endothelial barrier function independently of Orail and Ca2+ entry. Sci Signal. 2013;6(267):ra18.

40. Tiruppathi C, et al. Role of Ca2+ signaling in the regulation of endothelial permeability. Vascul Pharmacol. 2002;39(4-5):173-185.

41. Hartzell CA, et al. Calcium influx through CRAC channels controls actin organization and dynamics at the immune synapse. Elife. 2016;5:e14850.

42. Smith KM, et al. Structural basis for importin- $\alpha$ binding of the human immunodeficiency virus tat. Sci Rep. 2017;7(1):1650.

43. Aguilera TA, et al. Systemic in vivo distribution of activatable cell penetrating peptides is superior to that of cell penetrating peptides. Integr Biol (Camb). 2009;1(5-6):371-381

44. Sarko D, et al. The pharmacokinetics of cell-penetrating peptides. Mol Pharm. 2010;7(6):2224-2231.

45. Su G, et al. Absence of integrin $\alpha v \beta 3$ enhances vascular leak in mice by inhibiting endothelial cortical actin formation. Am $J$ Respir Crit Care Med. 2012;185(1):58-66.

46. Zhao Y, et al. Protection of LPS-induced murine acute lung injury by sphingosine-1-phosphate lyase suppression. Am J Respir Cell Mol Biol. 2011;45(2):426-435.

47. Lucas R, et al. Protein kinase C- $\alpha$ and arginase I mediate pneumolysin-induced pulmonary endothelial hyperpermeability. Am $J$ Respir Cell Mol Biol. 2012;47(4):445-453.

48. Wilson MR, et al. Differential roles of p55 and p75 tumor necrosis factor receptors on stretch-induced pulmonary edema in mice. Am J Physiol Lung Cell Mol Physiol. 2007;293(1):L60-L68.

49. Markowicz P, et al. Multicenter prospective study of ventilator-associated pneumonia during acute respiratory distress syndrome. Incidence, prognosis, and risk factors. ARDS Study Group. Am J Respir Crit Care Med. 2000;161(6):1942-1948.

50. Proudfoot A, et al. Novel anti-tumour necrosis factor receptor-1 (TNFR1) domain antibody prevents pulmonary inflammation in experimental acute lung injury. Thorax. 2018;73(8):723-730.

51. Wilson MR, et al. Inhibition of TNF receptor p55 by a domain antibody attenuates the initial phase of acid-induced lung injury in mice. Front Immunol. 2017;8:128.

52. Hordijk PL. Regulation of NADPH oxidases: the role of Rac proteins. Circ Res. 2006;98(4):453-462.

53. Ridley AJ. Rho GTPases and cell migration. J Cell Sci. 2001;114(Pt 15):2713-2722.

54. Rougerie P, et al. Generation of membrane structures during phagocytosis and chemotaxis of macrophages: role and regulation of the actin cytoskeleton. Immunol Rev. 2013;256(1):222-239.

55. Ashino Y, et al. $[\mathrm{Ca}(2+)](\mathrm{i})$ oscillations regulate type II cell exocytosis in the pulmonary alveolus. Am J Physiol Lung Cell Mol Physiol. 2000;279(1):L5-L13.

56. Hook JL, et al. Disruption of staphylococcal aggregation protects against lethal lung injury. J Clin Invest. 2018;128(3):1074-1086.

57. Lindert J, et al. Chloride-dependent secretion of alveolar wall liquid determined by optical-sectioning microscopy. Am J Respir Cell Mol Biol. 2007;36(6):688-696.

58. Wang PM, et al. Rapid alveolar liquid removal by a novel convective mechanism. Am J Physiol Lung Cell Mol Physiol. 2001;281(6):L1327-L1334.

59. Riedl J, et al. Lifeact: a versatile marker to visualize F-actin. Nat Methods. 2008;5(7):605-607.

60. Lewis JF, et al. Altered surfactant function and metabolism in rabbits with acute lung injury. J Appl Physiol (1985). 1990;69(6):2303-2310.

61. Brackenbury AM, et al. Evaluation of alveolar surfactant aggregates in vitro and in vivo. Eur Respir J. 2002;19(1):41-46.

62. Puligandla PS, et al. Alveolar environment influences the metabolic and biophysical properties of exogenous surfactants. $J$ Appl Physiol (1985). 2000;88(3):1061-1071.

63. Selinger SL, et al. Distribution volumes of [131I]albumin, [14C]sucrose, and 36Cl in sheep lung. J Appl Physiol. 1975;39(5):773-779. 